\title{
Traçabilité en assistance médicale à la procréation
}

\author{
L. LARUE
}

Centre de Traitement des Stérilités. Hôpital des Diaconesses, Paris

RÉSUMÉ

Une procédure de traçabilité en AMP permet de créer une trace écrite des contrôles effectués pour assurer une attribution exacte des gamètes ou des embryons lors des techniques d'insémination ou de fécondation in vitro. Elle impose une analyse des étapes à risques d'erreurs et la mise au point de systèmes de sécurité. Nous proposons dans ce travail quelques bases de réflexion sur ce sujet.

Mots Clés : Traçabilité, AMP, procédure, qualité, FIV.

\section{INTRODUCTION}

L'Assistance Médicale à la Procréation (AMP) recouvre les pratiques cliniques et biologiques permettant la conception in vitro, le transfert d'embryons, l'insémination artificielle, ainsi que toutes techniques d'effet équivalent, permettant la procréation en dehors du processus naturel.

Les actes cliniques correspondants sont: le recueil de gamètes par ponction, le transfert d'embryons, les inséminations avec sperme du conjoint ou avec sperme de donneur. Les actes biologiques incluent les traitements des gamètes, les fécondations in vitro et les conservations de gamètes et d'embryons.

Actuellement, la sécurité des actes d'AMP repose sur la conscience professionnelle de chaque intervenant et sur des procédures non spécifiques de biologie. Il n'existe pas de consensus ni de documents de traçabilité communs aux différents centres d'AMP.

La demande des couples traités, l'évolution médicale vers des démarches qualités, certification et accréditation, plaident pour la mise au point de procédures spécifiques de traçabilité en AMP. Nous livrerons ici quelques réflexions sur ce sujet qui fait l'objet d'un travail depuis 2 ans dans notre Centre.

\section{MÉTHODOLOGIE D'UNE PROCÉDURE DE TRAÇABILITÉ EN AMP}

Une procédure de traçabilité doit permettre d'établir un document écrit, lisible par un tiers, garantissant la conformité des actes réalisés à une procédure référente. Celle-ci doit :

- Définir les objectifs poursuivis et ses champs d'application.

- Analyser les différentes étapes élémentaires des techniques étudiées et en déduire les étapes à risques d'erreurs.

- Proposer des systèmes de sécurité visant à limiter les risques d'erreurs.

- Établir un document de synthèse.

Correspondance : Dr Lionel LARUE, Centre de Traitement des Stérilités. Hôpital des Diaconesses, Paris 75012. amp@ch-diaconesses.fr

Communication aux 3èmes Journées Nationales Assurance de Qualité en Biologie de la Reproduction, 26 $\& 27$ Octobre 2000, Nancy. 


\section{PROPOSITION DE PROCÉDURE DE TRAÇABILITÉ EN AMP}

1. OBJECTIF POURSUIVI : garantir une attribution exacte des gamètes ou des embryons lors des actes d'AMP en définissant un document écrit permettant de visualiser les contrôles des étapes définies comme à risques d'erreurs.

2. Champs d'application de la procédure : les actes cliniques et biologiques concernant les gamètes ou les embryons en AMP.

\section{ANALYSE DES ÉTAPES ÉLÉMENTAIRES DES ACTES D'AMP.}

- Insémination avec le sperme du conjoint (I.A.C.) :

Accueil de Monsieur X

Recueil du sperme dans un réceptacle par Monsieur X

Le réceptacle est confié par Monsieur X au biologiste du laboratoire de biologie de la reproduction

Prélèvement par le biologiste avec une pipette du sperme contenu dans le réceptacle

Transfert du sperme dans un tube à essai pour préparation

Transfert du sperme du tube à essai dans le cathéter de transfert

Transfert par le clinicien du sperme préparé chez Madame X

\section{- Fécondation in vitro (F.I.V.)}

Accueil de Monsieur X. Étapes identiques de préparation du sperme

Accueil de Madame X au bloc opératoire

Recueil des ovocytes par ponction échoguidée dans des seringues étiquetées et stockées dans une boite isotherme

Transfert de la boîte au laboratoire de biologie de la reproduction

Le jour même : recherche des ovocytes par transfert du contenu de chaque seringue dans une boîte de Pétri. Isolement de chaque ovocyte dans un puit (boîte à quatre puits). Prélèvement du sperme de Monsieur X à l'aide d'une pipette et mise en contact avec l'ovocyte dans le puit. Transfert de la boîte dans une étuve.
Le lendemain : transfert de chaque ovocyte dans une boîte de Pétri pour décoronisation puis mise en culture dans un milieu frais dans une nouvelle boîte à quatre puits. Transfert de la boîte dans l'étuve.

Le surlendemain : renouvellement du milieu et de la boîte à quatre puits. Repérage puis chargement des embryons dans un cathéter de transfert.

Le cathéter de transfert est remis par le biologiste au clinicien.

Le clinicien réalise le transfert des embryons de Madame et Monsieur X chez Madame X.

\section{IDENTIFICATION DES ÉTAPES ClÉS, SOURCES D'ERREURS.}

Trois types d'erreurs lors du parcours d'une AMP sont théoriquement possibles :

Type 1 : Erreur de procédure technique. Il s'agit du non-respect d'une procédure type déjà connue, par exemple : contamination d'un sperme par un autre sperme lors de la préparation liée à l'oubli du changement d'un cône de pipette.

Type 2 : Erreur d'identification d'un membre du couple. ( $\otimes$ dans la figure) aboutissant à une erreur d'attribution des gamètes ou des embryons.

Type 3 : Erreur d'identification d'un prélèvement. ( dans la figure) entraînant la même conséquence.

Les erreurs de types 2 et 3 sont à redouter essentiellement lors des changements d'intervenants (passage d'un prélèvement d'un clinicien à un biologiste ou l'inverse) ou des changements de supports des gamètes ou des embryons. Les étapes à risque maximal sont celles qui cumulent les deux types de changements, par exemple le transfert embryonnaire ou les embryons sont transférés d'une boîte à quatre puits dans un cathéter de transfert et confiés au clinicien par le biologiste.

L'identification des étapes à risques d'erreurs de type 2 et 3 est résumée dans la figure 1 .

\section{Proposition de systèmes de sécurité des ACTES D'AMP}


Figure 1. Représentation schématique des procédures et des risques d'erreur en AMP.

Risque d'erreur d'identification d'un membre du couple : $\otimes$;

Risque d'erreur d'identification d'un prélèvement : • .

CLINIQUE

Médecin, Infirmières
BIOLOGIE

Biologiste, Techniciens

\section{CLINIQUE}

Médecin

\section{I.A.C. :}

Accueil de $\mathrm{Mr}$ recueil de sperme

$\longrightarrow$ Préparation

$\otimes$ réceptacle

Tube à essai

Accueil de Mme

Insémination

$\otimes$

$\otimes$

\section{F.I.V. :}

Accueil de $\mathrm{Mr}$ recueil de sperme

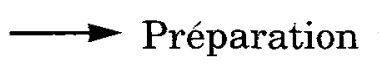
sperme

$\otimes$ réceptacle

Tube à essai préparé

Accueil de Mme Ponction Follicules Recherche ovocytes $\otimes$ Seringues Boite de Pétri

Insémination

Boite à quatre puits

Culture

Embryons $\rightarrow$ cathéter $\rightarrow$ Réimplantation $\otimes$ 
- Les erreurs de type 1 peuvent être limitées par la définition de procédures consensuelles des actes d'AMP. Celles-ci font actuellement l'objet de réunions régulières, de rédaction d'un guide de bonnes pratiques et de réflexion de groupe de travail parmi les professionnels de l'AMP [1].

- Les erreurs de types 2 peuvent être limitées par la mise en place d'une procédure type de contrôle des identités des membres d'un couple aboutissant à une trace écrite pour chaque étape à risque soit : le recueil ovocytaire, le recueil de sperme, l'insémination ou le transfert embryonnaire.

- Les erreurs de type 3 peuvent être prévenues par le contrôle des étapes de changement de supports des gamètes ou des embryons. Un double contrôle des étapes clés peut être réalisé par deux personnes différentes, la poursuite de la procédure n'est alors possible qu'après validation écrite du contrôle. Une automatisation et une informatisation de ces contrôles est une autre possibilité qui est plus simple à utiliser au quotidien, mais qui demande la mise au point d'un étiquetage adapté, résistant aux conditions thermique et hygrométrique de conservation des gamètes et des embryons, d'un système de lecture de ces étiquettes et d'un programme de gestion des données informatisées.

- Un document de suivi des personnes et des actes effectués doit être créé. Ce document peut prendre la forme d'une fiche navette suivant le couple de l'accueil jusqu'à l'insémination ou la réimplantation des embryons où chaque étape clé fait l'objet d'une identification et d'une validation qui conditionne la poursuite du parcours.

\section{CONCLUSION}

Les procédures de traçabilité sont nécessaires pour matérialiser la qualité des contrôles réalisés lors des actes d'AMP. Il est souhaitable que les professionnels de l'AMP en déterminent les modalités afin d'établir un système efficace dont la mise en œuvre n'alourdisse pas inutilement les procédures actuelles.

\section{RÉFÉRENCES}

GIANAROLI L., PLACHOT M., MANDELBAUM J., SELVA J. et al. : ESHRE guidelines for good practice in IVF laboratories. Hum. Reprod., 2000, 15 : 2241-2246.

\section{ABSTRACT \\ Traceability in assisted reproductive technology}

\section{LARUE}

An ART traceability procedure provides a written record of the controls performed to ensure precise allocation of gametes or embryos during insemination or IVF techniques. This procedure requires analysis of the various steps associated with a risk of error and optimization of safety systems. The author provides a basis for further thought on this subject.

Key words: Traceability, ART, Procedure, Quality, IVF 